\title{
A New Technique for Oral Endotracheal Airway Access in Rabbits under Fluoroscopic Control: An Easy Way for Drug Administration in Chronic Experiments
}

\author{
W. Kersjes G. Hildebrandt K. Schunk R. König A. Berlis \\ H. Schild \\ Department of Radiology, University of Bonn, Germany
}

\begin{abstract}
Endotracheal drug administration plays an important role in rabbit models of pulmonary injury and lung disease. However, anatomical features of the oral fissure and pharynx of rabbits make orotracheal intubation a difficult task with a high failure rate. We developed a new technique for airway access in rabbits under fluoroscopic control using a balloon catheter. The procedure consists of four major steps: (1) a shortened 5-french vertebralis catheter is placed into the mesopharynx just above the epiglottic valve, (2) through the catheter lumen a guidewire is carefully moved across the epiglottis and inserted into the trachea, (3) the vertebralis catheter is replaced by a balloon catheter that subsequently is introduced into the tracheal
\end{abstract}

tree over the guidewire, and (4) with the balloon catheter in the right position the guidewire is removed and the balloon is inflated so that finally the drug can be delivered. This procedure was performed in $55 \mathrm{New}$ Zealand White rabbits under general anesthesia with ketamine/xylazine. In all 55 cases the procedure was performed successfully without any major complications and within a reasonably short time period. Removal of the catheter and awakening of the animals were uneventful. The described technique is a simple and safe method for airway access in rabbits and can be useful in chronic experiments for endotracheal drug administration.

\section{Introduction}

Endotracheal drug administration plays an important role in rabbit models of pulmonary injury and lung disease. However, because of the narrow mouth, the relatively thick tongue, 
the upper incisors and because of the presence of sagittal niches located along both sides of the vocal cords getting airway access is known to be a difficult task. Various techniques for airway access have been described. The standard technique of orotracheal intubation has been successfully modified by different groups [1-6]. In acute animal experiments airway access may also be gained by tracheostomy. However, in chronic experimental studies tracheostomy can present difficulties. Although these difficulties can be avoided partially by using retrograde orotracheal intubation [7] this procedure still causes direct trauma to the cervical soft tissue and to the trachea.

We introduce a new technique for airway access and endotracheal drug administration in rabbits. The technique we used is related to the well-known Seldinger technique often used in interventional radiology.

\section{Material and Methods}

55 New Zealand White rabbits weighing between 2.5 and $3.5 \mathrm{~kg}$ were studied. All procedures were performed after initial administration of 1-2 ml general anesthesia [4 $\mathrm{ml}$ ketamine $(50 \mathrm{mg} / \mathrm{ml}), 2.5 \mathrm{ml}$ xylazine $(0.02 \mathrm{mg} / \mathrm{ml}), 0.9 \% \mathrm{NaCl}$ ad $50 \mathrm{ml}]$ into an ear vein, followed by repeated injection of $0.2-0.5 \mathrm{ml}$ to maintain anesthesia as needed. Fluoroscopy was used for monitoring and documentation of each step during the procedure.

After the onset of general anesthesia the animals were placed in a lateral decubitus position their neck being overextended. Their mouth was opened gently and a 5 -french vertebralis catheter that had been shortened to a length of $25 \mathrm{~cm}$ was placed into the mesopharynx just above the epiglottic valve. Through the catheter lumen a Terumo ${ }^{\circledR}$ guidewire was introduced. Mostly the guidewire first entered the proximal esophagus (fig. 1). In this case the catheter was also slid into the esophagus and the whole system was subsequently drawn back with the curved tip of the guidewire pointing to the front. The correct position of the system immediately proximal to the epiglottic valve was indicated by a sudden ventral movement of the tip of the guidewire (fig. 2). Careful movements of the guidewire tip made the animals swallow due to contact with the pharyngeal mucosa. While swallowing the guidewire could be carefully moved forward across the epiglottis and inserted into the trachea. After correct placement of the guidewire into the trachea the catheter was also advanced into the trachea (fig. 3). Subsequently the animals were rotated into a prone position and the Terumo guidewire was replaced by a thinner guidewire with a very soft distal tip (Terumo guidewire with a diameter of $0.018 \mathrm{inch}, 0.46 \mathrm{~mm}$. This guidewire could be placed into the distal tracheal tree without any difficulties and without causing injuries to the tracheobronchial tree or lung parenchyma. Finally the 5french vertebralis catheter was exchanged for a balloon catheter which was introduced into either the right or left lower lobe bronchus over the guidewire. After correct positioning of the balloon catheter the guidewire was removed and the balloon was inflated with a small amount of contrast medium (Solutrast ${ }^{\circledR} 300$ ) (fig. 4). Now the drug could be administered.

In our study either bleomycin sulfate $(10 \mathrm{mg} / \mathrm{kg}$ body weight) diluted in $5 \mathrm{ml} 0.9 \% \mathrm{NaCl}$ solution ( $\mathrm{n}=$ $44)$, or $5 \mathrm{ml}$ of pure $0.9 \% \mathrm{NaCl}$ solution $(\mathrm{n}=11)$ were slowly delivered to the lung. After the application animals were rotated carefully in order to get a homogeneous distribution of the instilled agent. During the whole procedure the balloon was kept inflated to delimit the area of the involved lung tissue from the remaining healthy uninvolved lung parenchyma. During the removal of the balloon catheter and for the next 5-10 min the animals were kept in a left or right recumbent position depending on the side into which the agent was injected. After complete awakening from anesthesia the animals were put back into their box.

Finally, the animals were killed with $20 \mathrm{ml}$ of ketamine/xylazine [ $4 \mathrm{ml}$ ketamine $(50 \mathrm{mg} / \mathrm{ml}), 2.5 \mathrm{ml}$ xylazine $(0.02 \mathrm{mg} / \mathrm{ml}), 0.9 \% \mathrm{NaCl}$ ad $50 \mathrm{ml}$ ) plus $20 \mathrm{ml}$ potassium hydrochloride solution $(1 \mathrm{~mol} / \mathrm{ml})$ at different time intervals ( 3 and $24 \mathrm{~h}, 8,14,30$ and 80 days) after intrabronchial application of the agents. The lungs were removed and a fixative solution of $10 \%$ formalin was infused into the trachea under the pressure of $10 \mathrm{~cm} \mathrm{H}_{2} \mathrm{O}$. After being immersed in the fixative solution for 4 weeks, the lungs were cut into $10-\mathrm{mm}$ slices and certain areas were selected for histological studies after hematoxylin-eosin and van Gieson/elastica staining. 


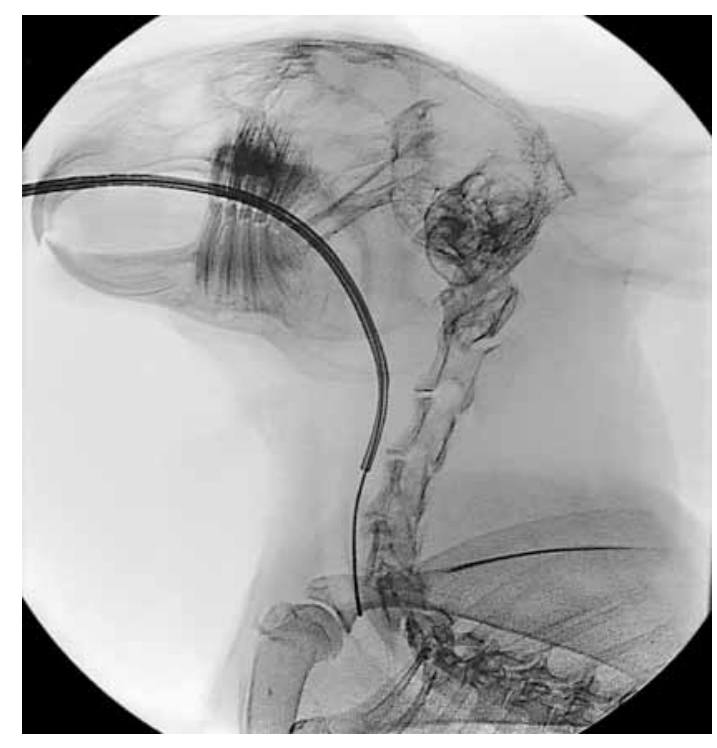

Fig. 1. Guidewire and catheter are positioned in the proximal esophagus.

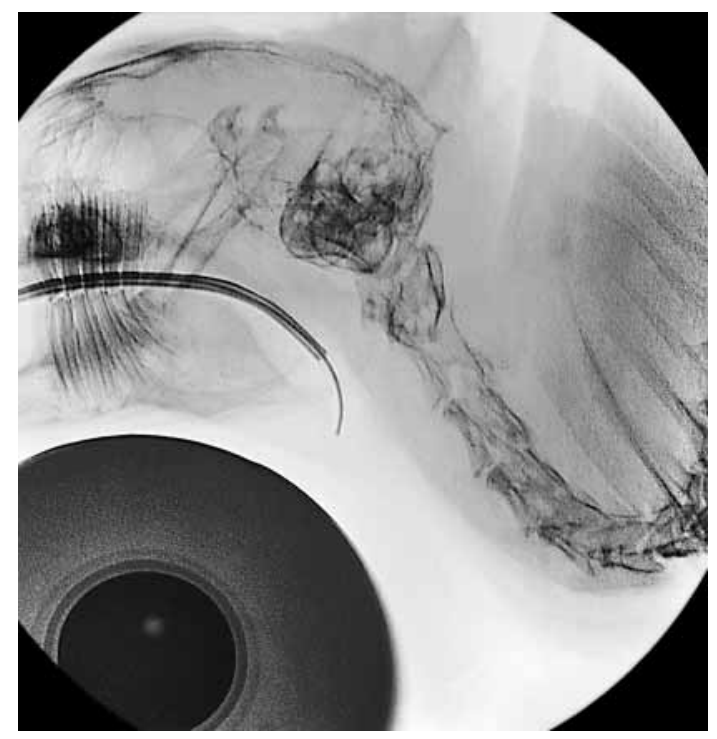

Fig. 2. Retraction of catheter and guidewire into the larynx immediately above the epiglottic valve with the tip of the guidewire pointing to the front.

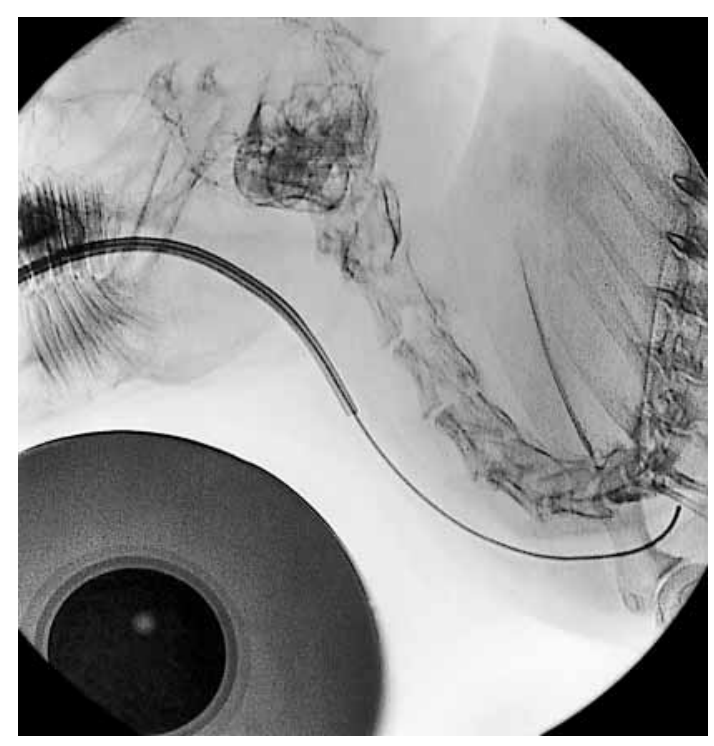

Fig. 3. Introduction of guidewire and catheter into the trachea.

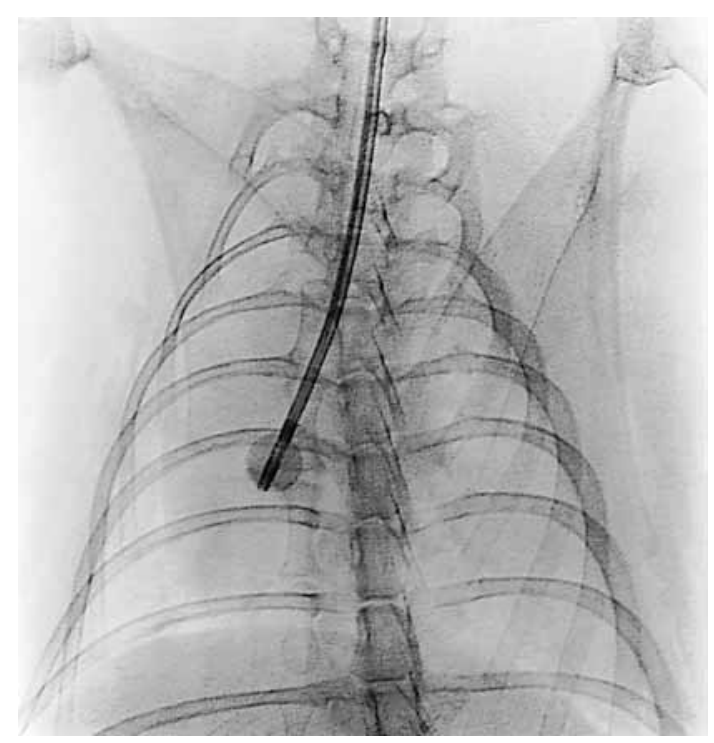

Fig. 4. Inflated balloon catheter(with contrast medium) in the right main bronchus for selective and safe intrabronchial drug administration. 


\section{Results}

In all 55 cases endotracheal or endobronchial placement of the balloon catheter and administration of the drug were performed successfully. On average, the procedures lasted between 10 and $15 \mathrm{~min}$ from the onset of general anesthesia until the drug could be delivered. Removal of the balloon catheter and awakening were uniformly uneventful. All animals tolerated the procedures very well without major complications or death.

Examination of specimens from trachea and lungs did not show any macroscopic injury due to the technique itself. At histologic evaluation lung tissue, which was altered secondarily to drug effects, could clearly be delimited from the remaining healthy uninvolved lung parenchyma. Without exception drug effects were restricted to the lung area that was demarcated by the inflated balloon.

\section{Discussion}

Airway access in rabbits is known to be a difficult task. Davies et al. [3] reported that the standard technique of orotracheal intubation is enormously facilitated by placing the operator behind the animal, viewing it from the dorsal surface of the head. Krüger et al. [5] recommended the use of a transparent tube in order to see respiration moisture with the tube in the correct location. Since the tube will obstruct the view of the vocal cords when introduced into the channel of the laryngoscope, Bechtold and Abrutyn [2] developed a way of side placement of the endotracheal tube into the rabbit mouth that permitted visualization of the laryngeal structures. The same problem was overcome by Macrae and Guerreiro [6] who inserted a tube which contained a catheter extending beyond the opening and into the trachea. Blind intubation without the use of a laryngoscope was performed by Alexander and Clark [1] and by Fick and Schalm [4]. Their results indicate that the narrowness of the oropharynx lowers the chances of misplacing the tube into the esophagus. In acute animal experiments tracheostomy provides an alternative method for airway access. However, in chronic experimental studies the suturing of a tracheostoma is difficult and can be hazardous. These difficulties can be avoided partially by using retrograde orotracheal intubation. Corleta et al. [7] described a modification of the retrograde orotracheal intubation technique with the introduction of an orotracheal tube over a guidewire. The guide was inserted into the trachea via cervicotomy and delivered through the mouth. However, this procedure still causes direct trauma to the cervical soft tissue and to the trachea.

In comparison to the technique described above our method of getting airway access and of endotracheal drug administration has been shown to be very reliable, simple and safe. The placement of the balloon catheter could be performed without any trauma to the oropharyngeal mucosa, the trachea or the distal tracheal tree. However, the use of a guidewire with a very soft tip is strongly recommended for the prevention of injuries. It is also important not to increase force if the forward movement of guidewires or catheters becomes more difficult. A repeated careful movement back and forward is often helpful in this situation. All procedures should be done under fluoroscopy. The use of contrast medium for balloon inflation allows for the early detection of complications like incorrect catheter position, for example complete occlusion of airways due to an incorrect position of the inflated balloon in the trachea. The balloon is an easy and very efficient tool to keep the catheter in place, to control the regional distribution of the injected drug and, there- 
fore, to prevent injury to the adjacent lung parenchyma. Whereas other techniques make use of readily available equipment and can be performed in nearly any laboratory [1-5], the need for specific catheters and guidewires as well as the use of fluoroscopy are the disadvantages of the technique used in this study. However, the easy performance, the reason- ably short time period needed, the lack of complications and the well-controlled distribution of the administered drug suggest that the described technique provides an excellent way of endotracheal drug administration in rabbit models of pulmonary injury and lung disease.

\section{References}

1 Alexander DJ, Clark GC: A simple method of oral endotracheal intubation in rabbits. Lab Anim Sci 1980; 30:871-873.

2 Bechtold SV, Abrutyn D: An improved method of endotracheal intubation in rabbits. Lab Anim Sci 1991;41:630-631.
3 Davies A, Dallak M, Moores C: Oral endotracheal intubation of rabbits (Oryctolagus cuniiculus). Lab Anim 1996;30:182-183.

4 Fick TE, Schalm SW: A simple technique for endotracheal intubation in rabbits. Lab Anim 1987;21:265266.

5 Krüger J, Zeller W, Schottmann E: A simplified procedure for endotracheal intubation in rabbits. Lab Anim 1994;28:176-177.
6 Macrae DJ, Guerreiro D: A simple laryngoscopic technique for the endotracheal intubation of rabbits. Lab Anim 1989;23:59-61.

7 Corleta O, Habazettl H, Kreimeier U, Vollmar B: Modified retrograde orotracheal intubation technique for airway access in rabbits. Eur Surg Res 1992;24:129-132. 